\title{
Order-parameter symmetries of domain walls in ferroelectrics and ferroelastics
}

\author{
Pierre Tolédano, ${ }^{1}$ Mael Guennou, ${ }^{2,{ }^{*}}$ and Jens Kreisel ${ }^{2,3}$ \\ ${ }^{1}$ Laboratoire des Systèmes Complexes, Université de Picardie, 80000 Amiens, France \\ ${ }^{2}$ Département Science et Analyse des Matériaux, CRP Gabriel Lippmann, 41 rue du Brill, L-4422 Luxembourg \\ ${ }^{3}$ Physics and Materials Science Research Unit, University of Luxembourg, 41 Rue du Brill, L-4422 Belvaux, Luxembourg
}

(Received 22 January 2014; revised manuscript received 31 March 2014; published 16 April 2014)

\begin{abstract}
The symmetry of boundaries between ferroelectric, ferroelastic, and antiphase domains is a key element for a theoretical understanding of their properties. Here, we derive this symmetry from their organic relation to the symmetry of the primary transition order parameters. The domain wall symmetries are shown to coincide with directions of the order-parameter $n$-dimensional vector space, corresponding to sum of the vectors associated with adjacent domain states. This property is illustrated by the determination of the maximal symmetries of domain walls in $\mathrm{BaTiO}_{3}, \mathrm{LaAlO}_{3}, \mathrm{SrTiO}_{3}$, and $\mathrm{Gd}_{2}\left(\mathrm{MoO}_{4}\right)_{3}$. Besides, the domain pattern in $\mathrm{YMnO}_{3}$ is interpreted as resulting from an annihilation-creation process, the annihilation of the antiphase domain walls creating six ferroelectric domain walls merging at a single point.
\end{abstract}

DOI: 10.1103/PhysRevB.89.134104

PACS number(s): 77.80.Dj

\section{INTRODUCTION}

Multiferroic materials, i.e., materials possessing simultaneously several ferroic orders such as ferromagnetism, ferroelectricity, and/or ferroelasticity, currently attract a great deal of interest because of their intriguing coupling phenomena. One of the interesting and intrinsic properties of ferroic materials is the presence of domains separated by domain walls (DWs, also called domain boundaries) [1]. DWs can be seen as spatially extended transition regions mediating the change in the ferroic order parameters from one domain to another with resulting gradient effects. For a long time, however, ferroelectric and ferroelastic DWs have been considered experimentally as interfaces with negligible thickness. It is only within the last few years, thanks to now available atomic-resolution studies such as high-resolution transmission electron microscopy, various atomic force microscopies (c-AFM, PFM), etc. that their real complexity has been revealed. This has inspired a new paradigm of ferroic devices where the domain wall, rather than the domain, is the active element. It has been argued that the exploitation of the small size of domain walls (of the order of several nanometers) and their different functional properties present a high potential towards domain-wall nanoelectronics [2-4].

Experimentally, pioneering work on $\mathrm{WO}_{3}$ [5] has shown that ferroelastic twin walls exhibit electric conductivity or even superconductivity. Conducting domain walls were observed in several materials, e.g., $\mathrm{BiFeO}_{3}$ [6] or $\mathrm{BaTiO}_{3}$ [7], which exhibit electronic conductivity up to $10^{9}$ higher than the insulating domains. Interestingly, in other ferroelectric materials such as in $\mathrm{LiNbO}_{3}$ single crystals, the domain-wall conductivity is induced only by super-band-gap UV-light illumination [8]. Ferroelastics such as $\mathrm{CaTiO}_{3}$ [9] or $\mathrm{SrTiO}_{3}$ [10] present the striking example of a domain wall with polar properties while the bulk material is not ferroelectric. The full understanding and engineering of such domain-wall properties remain to be established. In particular, it is not yet clear if all reported results reflect the intrinsic properties of the domain walls,

*guennou@lippmann.lu or if they are related to the complex minimization of the different energy ingredients, such as surface energy, residual stresses, shape anisotropy, structural defects, impurities, or stoichiometry issues contributing to their stabilization. As a consequence of this, theoretical tools are needed to distinguish intrinsic from extrinsic effects and properties, symmetry being thereby an essential ingredient. The symmetry properties of DW have already been subjected to theoretical investigations. Classically, the symmetry of the domain wall can be deduced by combining the common symmetry elements of the adjacent domains plus additional symmetries transforming one domain into another [11]. Notably, following this approach, the symmetries of strain-compatible walls in ferroelectrics and ferroelastics has been determined and tabulated [11,12].

Here, we propose an alternative approach which allows deriving the symmetry of the domain walls from their organic relationship with the primary transition order parameter, giving rise to the domain pattern. We show that although in most cases the symmetry of domain walls as obtained by geometrical considerations [11] can be straightforwardly deduced from the symmetry of the corresponding order parameter, in a number of specific situations, considering or ignoring the order-parameter symmetry leads to different predictions.

\section{GENERAL APPROACH}

Our proposed approach for determining domain-wall symmetries is based on the following property: The symmetry of the domain wall between two adjacent domains associated with the vectors $\vec{V}_{1}$ and $\vec{V}_{2}$ in the $n$-dimensional order-parameter vector space is an isotropy subgroup of the symmetry group of the domain state corresponding to $\vec{V}_{1}+\vec{V}_{2}$. This is inferred from the fact that the symmetry group of a domain wall leaves by definition the domain pair invariant: the common symmetry operations of adjacent domains represented by $\vec{V}_{1}$ and $\vec{V}_{2}$ as well as the operations exchanging the two vectors also leave their sum invariant. Therefore, the symmetry group $G_{0}$ of $\vec{V}_{1}+\vec{V}_{2}$ contains the isotropy subgroup $G$ corresponding to the symmetry of the domain wall. $G$ may coincide with $G_{0}$ or be a proper isotropy subgroup of $G_{0}$. 
The symmetry $G$ of the domain wall as determined from our approach is described by a point group. It is a maximal symmetry that is independent of a particular choice of domainwall orientation, which in practice may result from elastic compatibility, from the history and preparation of the sample or the minimization of bound charges. A particular choice of orientation requires us to take into account the planar character of the wall in space and describe the DW symmetry by a layer group [11]. A symmetry lowering generally occurs in the process, which is linked to the geometric arrangement of domains in space and the DW orientation but does not follow from the phase transition mechanism itself. Besides, when assuming a finite wall thickness, the DW symmetry may be further reduced if a phase transition occurs within the DW, with the emergence of another stable state associated with the same order parameter inducing the transition in the bulk [13]. Such structural phase transitions have not yet been observed in the domain walls of ferroelectric or ferroelastic materials but have been predicted theoretically [14-18] and reported experimentally in domain walls separating magnetic domains [19-21]. Such considerations, which are inherent to the metastable nature of domains and domain walls due to their positive energy, concern a marginal number of experimental situations and do not limit the interest of our general approach.

In the following, we shall apply this method to model systems representative of the different situations occurring in ferroelectric and ferroelastics: (i) the proper ferroelectric $\mathrm{BaTiO}_{3}$, (ii) the ferroelastics $\mathrm{SrTiO}_{3}$ and $\mathrm{LaAlO}_{3}$, and (iii) the improper ferroelectrics $\mathrm{Gd}_{2}\left(\mathrm{MoO}_{4}\right)_{3}$ and $\mathrm{YMnO}_{3}$.

\section{DOMAIN-WALL SYMMETRY IN FERROELECTRIC BaTiO}

As a first illustrative example of our approach, we consider the ferroelectric domains of the three ferroelectric-ferroelastic phases of barium titanate $\mathrm{BaTiO}_{3}$ having the $4 \mathrm{~mm}, 3 \mathrm{~m}$, and $m m 2$ point groups [22]. Table I lists the set of stable states associated with the primary polarization order parameter and the corresponding symmetries of each domain state $[23,24]$. Figure 1(a) shows the vectors associated with one domain of each state in the three-dimensional order-parameter space $E_{3}$.

TABLE I. Equilibrium states induced by the three-component polarization order parameter associated with the ferroelectric transitions in $\mathrm{BaTiO}_{3}$ and the improper ferroelastic transitions in $\mathrm{LaAlO}_{3}$ and $\mathrm{SrTiO}_{3}$ : labeling of the state, equilibrium values of the orderparameter components for one domain state, space group of the domain state.

\begin{tabular}{lccccc}
\hline \hline & $\mathrm{BaTiO}_{3}$ & & \multicolumn{3}{c}{$\mathrm{SrTiO}_{3}, \mathrm{LaAlO}_{3}$} \\
\hline 0 & $(0,0,0)$ & $P m \overline{3} m$ & 0 & $(0,0,0)$ & $P m \overline{3} m$ \\
$\mathrm{I}$ & $(P, 0,0)$ & $\mathrm{P} 4 m m$ & $\mathrm{I}$ & $(\eta, 0,0)$ & $\mathrm{I} 4 / m c m$ \\
II & $(P, P, 0)$ & $\mathrm{A} m m 2$ & II & $(\eta, \eta, 0)$ & Imma \\
III & $(P, P, P)$ & $R 3 m$ & III & $(\eta, \eta, \eta)$ & $R \overline{3} c$ \\
IV & $\left(P_{x}, P_{x}, P_{z}\right)$ & $P m$ & IV & $\left(\eta_{1}, \eta_{2}, 0\right)$ & $C 2 / m$ \\
V & $\left(P_{x}, P_{y}, 0\right)$ & $C m$ & V & $\left(\eta_{1}, \eta_{1}, \eta_{1}\right)$ & $C 2 / c$ \\
VI & $\left(P_{z}, P_{z}, P_{x}\right)$ & $C m$ & VI & $\left(\eta_{3}, \eta_{3}, \eta_{1}\right)$ & $C 2 / m$ \\
VII & $\left(P_{x}, P_{y}, P_{z}\right)$ & $P 1$ & VII & $\left(\eta_{1}, \eta_{2}, \eta_{3}\right)$ & $P \overline{1}$ \\
\hline \hline
\end{tabular}

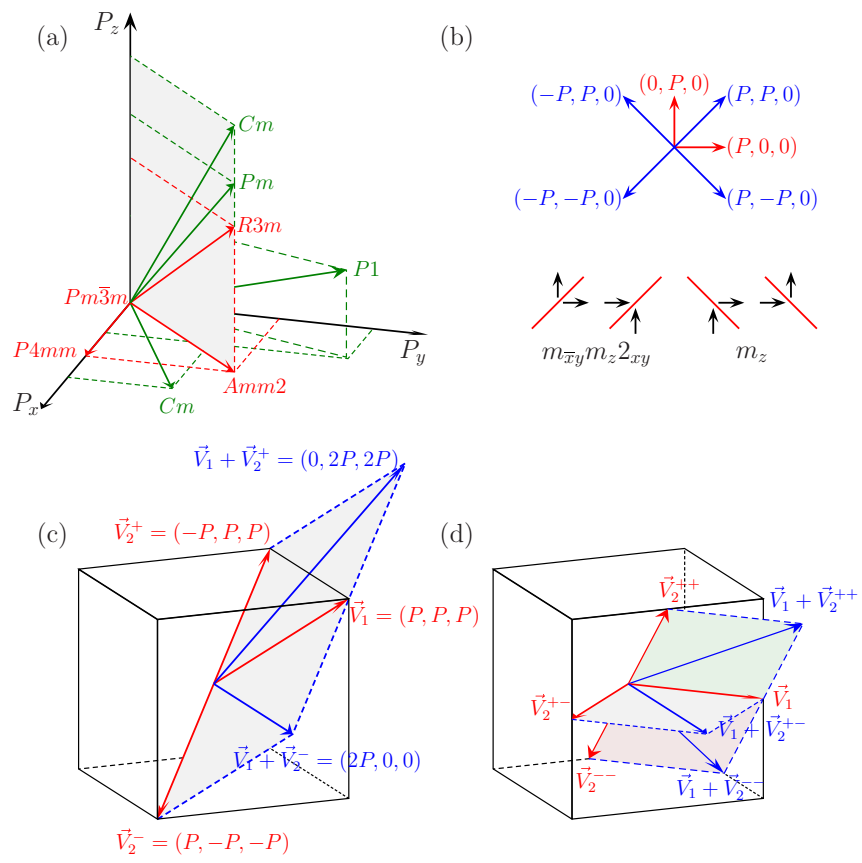

FIG. 1. (Color online) (a) Directions representing one domain of the equilibrium states induced by the three-dimensional order parameter of $\mathrm{BaTiO}_{3}$. Red arrows correspond to the observed states. (b)-(d) Vectors associated with domain walls (blue arrows) between adjacent domains (red arrows) in the tetragonal (b), rhombohedral (c), and orthorhombic (d) phases of $\mathrm{BaTiO}_{3}$. The detailed meaning of the figures is in the text. In (b), black arrows show the orientations of the polarization on both sides of domain walls.

In the tetragonal phase the sum of the vectors of $E_{3}$ associated with a pair of ferroelectric domains at $90^{\circ}$ coincides with the symmetry of the orthorhombic domain states. Choosing, e.g., $\vec{V}_{1}^{ \pm}=( \pm P, 0,0)$ and $\vec{V}_{2}^{ \pm}=(0, \pm P, 0)$, we have four possible combinations and two equivalent point-group symmetries, $m_{\bar{x} y} m_{z} 2_{x y}$ and $m_{x y} m_{z} 2_{\bar{x} y}$, corresponding to the symmetry of $\vec{V}_{1}^{ \pm}+\vec{V}_{2}^{ \pm}=( \pm P, \pm P, 0)$ (Table I). Depending on the orientation of the domain wall, each sum can yield the four configurations (head-to-head, tail-to-tail, tail-to-head and head-to-tail) observed experimentally [25], as illustrated in Fig. 1(b) for the $(P, P, 0)$ case. For head-to-tail configurations, the symmetry reduces from $m_{\bar{x} y} m_{z} 2_{x y}$ to $m_{z}$.

The domain-wall symmetries separating the $71^{\circ}$ and $109^{\circ}$ rhombohedral domains of $\mathrm{BaTiO}_{3}$ are obtained in the same way: putting $\vec{V}_{1}=(P, P, P)$ and $\vec{V}_{2}^{+}=(-P, P, P)$, the sum $\vec{V}_{1}+\vec{V}_{2}^{+}=(0,2 P, 2 P)$ corresponds to the orthorhombic domain state of symmetry $m_{x} m_{\bar{y} z} 2_{y z}$, providing the $71^{\circ}$ domain-wall symmetry [Fig. 1(c)]. With $\vec{V}_{2}^{-}=(P,-P,-P)$, the sum $\vec{V}_{1}+\vec{V}_{2}^{-}=(2 P, 0,0)$ coincides with the direction of the tetragonal domain state of symmetry $4_{x} \mathrm{~mm}$, the $109^{\circ}$ domain-wall symmetry corresponding to $m_{y z} m_{\bar{y} z} 2_{x}$ [Fig. 1(c)], which is a maximal isotropy subgroup of $4_{x} \mathrm{~mm}$.

Neighboring orthorhombic domains present a variety of domain-wall symmetries. For the domain states $\vec{V}_{1}=(P, P, 0)$ and $\vec{V}_{2}^{+-}=(P,-P, 0)$, the sum $\vec{V}_{1}+\vec{V}_{2}^{+-}=(2 P, 0,0)$ is associated with the tetragonal domain $4_{x} \mathrm{~mm}$ [Fig. 1(d)], the domain wall having the maximal isotropy subgroup 
(a)

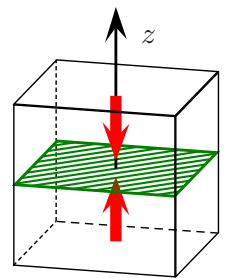

(d)
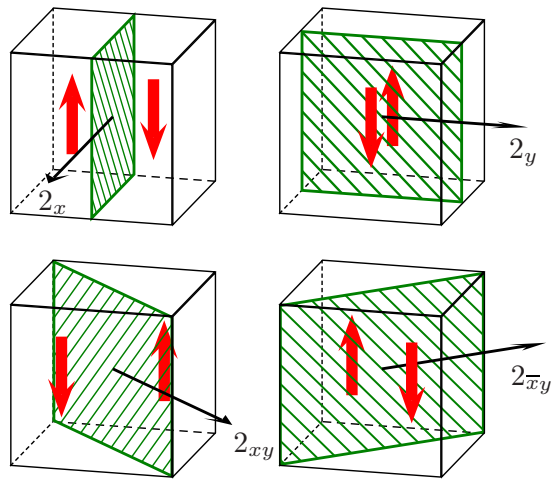

FIG. 2. (Color online) Orientation of the polarization (red arrows) on each side of the $180^{\circ}$ domain walls in the tetragonal [(a), (d)], rhombohedral (b), and orthorhombic (c) phases of $\mathrm{BaTiO}_{3}$. The detailed meaning of the figures is in the text.

$m_{y} m_{z} 2_{x}$. For $\vec{V}_{2}^{--}=(-P, 0,-P)$ the domain-wall symmetry $m_{x} m_{y z} 2_{\bar{y} z}$ is that of one of the 12 orthorhombic domain states $\vec{V}_{1}+\vec{V}_{2}^{--}=(0, P,-P)\left[\right.$ Fig. 1(d)]. For $\vec{V}_{2}^{++}=(P, 0, P)$ the sum $\vec{V}_{1}+\vec{V}_{2}^{++}=(2 P, P, P)$ yields a domain-wall symmetry $m_{\bar{y} z}$ corresponding to the monoclinic domain state denoted IV in Table I, which is not stabilized in bulk $\mathrm{BaTiO}_{3}$, although its presence has been disputed in literature [26].

For determining the domain-wall symmetries between the $180^{\circ}$ ferroelectric domains of $\mathrm{BaTiO}_{3}$, one has to take into account the reduction of the order-parameter space $E_{3}$ for a given orientation of the domains. Considering the tetragonal $180^{\circ}$ domains represented by the $E_{3}$ vectors $\vec{V}_{1}=(0,0, P)$ and $\vec{V}_{2}=(0,0,-P)$ gives the sum $\vec{V}_{1}+\vec{V}_{2}=(0,0,0)$, corresponding to the equilibrium value of the order parameter in the parent ferroelastic domain associated with $\vec{V}_{1}$ and $\vec{V}_{2}$, which has the tetragonal point-group symmetry $4_{z} / \mathrm{mmm}$. This is the actual symmetry of the head-to-head or tail-to-tail $180^{\circ}$ domain walls shown in Fig. 2(a). Following the same scheme, the domain wall separating $180^{\circ}$ rhombohedral antiparallel domains such as $\pm(P, P, P)$ has the symmetry $R \overline{3}^{x y z} m$ of the parent ferroelastic domain [Fig. 2(b)], whereas the orthorhombic ferroelastic domain symmetry $\mathrm{mmm}$ coincides with the symmetry of orthorhombic domain walls between $\pm(P, P, 0) 180^{\circ}$ domains [Fig. 2(c)].

For tetragonal $180^{\circ}$ domains separated by planes $\left(m_{x}, m_{y}, m_{x y}, m_{\bar{x} y}\right)$ containing the fourfold rotation $4_{z}$, the symmetry of the domain walls is lowered when fixing the orientation of one of the planes. This is consistent with our approach, since lowering the $m \overline{3} \mathrm{~m}$ symmetry to $4 / \mathrm{mmm}$ yields a decomposition of the order-parameter space $E_{3}=$ $E_{2}+E_{1}$. In the two-dimensional order-parameter space $E_{2}$, spanned by the bases $\vec{V}_{1}=( \pm P, 0), \vec{V}_{2}=(0, \pm P)$ or $\vec{V}_{1}=$ $( \pm P, \pm P), \vec{V}_{2}=( \pm P, \mp P)$, the sum $\vec{V}_{1}+\vec{V}_{2}$ provides the domain-wall orientations $\bar{x} y, x y, y$, and $x$ having the respective monoclinic symmetries $2_{x y} / m_{x y}, 2_{\bar{x} y} / m_{\bar{x} y}, 2_{x} / m_{x}$, and $2_{y} / m_{y}$. They correspond to the four $180^{\circ}$ domain configurations shown in Fig. 2(d). In the one-dimensional order-parameter space $E_{1}$, the basic vector $\vec{V}$ coincides with the single domain state $\pm P$ of tetragonal symmetry $4_{z} / \mathrm{mmm}$, which is the symmetry of the head-to-head and tail-to-tail domain walls [Fig. 2(a)].

\section{DOMAIN-WALL SYMMETRY IN FERROELASTIC $\mathrm{SrTiO}_{3} \mathrm{AND} \mathrm{LaAlO}_{3}$}

As a second example we describe the domain-wall symmetries separating the ferroelastic domains in $\mathrm{LaAlO}_{3}$ and $\mathrm{SrTiO}_{3}$ which undergo improper ferroelastic transitions [27,28], leading, respectively, to rhombohedral and tetragonal phases for different equilibrium values of the same three-dimensional order-parameter symmetry $\left(\eta_{1}, \eta_{2}, \eta_{3}\right)$ [23] (Table I). In the cell-doubling transition $\operatorname{Pm} \overline{3} m(\vec{a}, \vec{b}, \vec{c}) \rightarrow R \overline{3} c(\vec{a}+\vec{b}, \vec{b}+$ $\vec{c}, \vec{c}+\vec{a})$ of $\mathrm{LaAlO}_{3}$, the order parameter has an eightfold degeneracy corresponding (1) to the equilibrium values I: $(\eta, \eta, \eta)$, II: $(-\eta, \eta, \eta)$, III: $(\eta,-\eta, \eta)$, IV: $(\eta, \eta,-\eta)$ associated with four ferroelastic domains $\left(e_{x y}, e_{z x}, e_{y z}\right),\left(-e_{x y},-e_{z x}, e_{y z}\right)$, $\left(-e_{x y}, e_{z x},-e_{y z}\right)$, and $\left(e_{x y},-e_{z x},-e_{y z}\right)$; and (2) to the opposite values V:( $(-\eta,-\eta,-\eta)$, VI: $(\eta,-\eta,-\eta)$, VII: $(-\eta, \eta,-\eta)$, VIII: $(-\eta,-\eta, \eta)$ representing antiphase domains related to the loss of the cubic translations $\vec{a}, \vec{b}$, and $\vec{c}$ at the transition. The ferroelastic domain walls I-II, I-III, I-IV, II-III, II-IV, and III-IV display two types of symmetries: (1) orthorhombic $m_{x} m_{y z} m_{\bar{y} z}$ for the domain wall I-II, associated with the sum $\vec{V}_{\mathrm{I}}+\vec{V}_{\mathrm{II}}=(0,2 \eta, 2 \eta)$, coinciding with one of the domain states of the orthorhombic Imma phase (Table I); and (2) again orthorhombic $m_{x} m_{y} m_{z}$ for the domain wall II-III, given by $\vec{V}_{\text {II }}+\vec{V}_{\text {III }}=(0,0,2 \eta)$, which is a maximal isotropy subgroup of the domain state $I 4_{z} / \mathrm{mmm}$ (Table I). Antiphase domain walls, i.e., boundaries between distinct antiphase domains within the same ferroelastic domain, correspond to the I-V, II-VI, III-VII, and IV-VIII pairs displaying the $R \overline{3} m$ symmetry of the rhombohedral ferroelastic domain.

A similar description can be given for the ferroelastic and antiphase domain walls in the tetragonal $\operatorname{I4/mcm}(\vec{a}+$ $\vec{c}, 2 \vec{b}, \vec{c}-\vec{a}$ ) phase of $\mathrm{SrTiO}_{3}$. Thus, the walls between the three ferroelastic domains have the orthorhombic symmetries $m_{x y} m_{\bar{x} y} m_{z}, m_{x z} m_{\bar{x} z} m_{y}$, and $m_{y z} m_{\bar{y} z} m_{x}$, corresponding to the point groups of the orthorhombic domain states Imma (Table I), whereas the symmetries of the three distinct antiphase domain walls have the ferroelastic domain symmetries $4_{u} / \mathrm{mmm}(u=x, y, z)$.

This result calls for comments in light of the recent experimental observation of piezoelectric resonance below $80 \mathrm{~K}$ in $\mathrm{SrTiO}_{3}$ [10], interpreted as a signature of the polar character of the domain walls. In $\mathrm{SrTiO}_{3}$ and $\mathrm{LaAlO}_{3}$, the primary order-parameter symmetry associated to the tilt of octahedra always preserves the inversion center and therefore leads to domain walls with an intrinsic nonpolar character. In contrast, the standard geometric approach [11] states that all compatible ferroelastic walls are polar, irrespective of any ferroelectric instability. This apparent contradiction can be waived by recalling that this latter prediction is only related to 
the boundary conditions imposed on the internal domain-wall structure for a specific wall orientation but is not related to the phase-transition mechanism. If this mechanism becomes more complex, with electric polarization also involved as an order parameter, as it is often assumed in studies on $\mathrm{SrTiO}_{3}$, we expect that a possible polar character of the wall will show up in the intrinsic symmetry of the domain wall derived from the order-parameter description, thereby distinguishing $\mathrm{SrTiO}_{3}$ from ordinary ferroelastics.

\section{ANTIPHASE DOMAIN WALLS IN Gd $2\left(\mathrm{MoO}_{4}\right)_{3}$

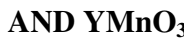

Although the domain-wall symmetry derived from the order-parameter symmetry often coincides with the symmetry resulting from the geometrical approach in terms of layer groups [11], such coincidence is not always realized, as, for example, at the improper ferroelectric-ferroelastic (FF) transition in gadolinium molybdate (GMO) [29]. The two-component order parameter $\left(\eta_{1}, \eta_{2}\right)$ describing the $P \overline{4} 2_{1} m(\vec{a}, \vec{b}, \vec{c}) \rightarrow$ $\operatorname{Pba} 2(\vec{a}-\vec{b}, \vec{a}+\vec{b}, \vec{c})$ transition in GMO has a fourfold degeneracy corresponding to the equilibrium domain states I: $\left(\eta_{1}, \eta_{2}\right)$, II: $\left(-\eta_{2}, \eta_{1}\right)$, III: $\left(-\eta_{1},-\eta_{2}\right)$, and IV: $\left(\eta_{2},-\eta_{1}\right)$, with two opposite FF domains $\pm\left(P_{z}, e_{x y}\right)$ [Fig. 3(a)]. There are four types of FF domain walls (I-II, II-III, III-IV, and I-IV) and two types of antiphase domain walls (I-III and II-IV). The four FF domains walls have the orthorhombic symmetry $m m 2$, which is higher than the geometrically determined monoclinic symmetry 2.

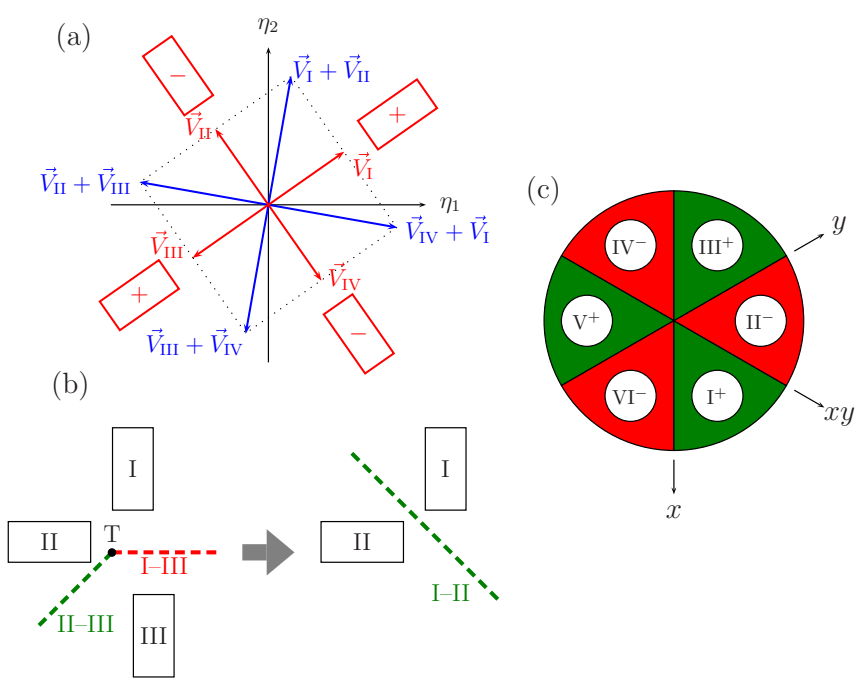

FIG. 3. (Color online) (a) Orientation of the FF domains (red arrows and rectangles) and domain-wall vectors (blue arrows) for the four equilibrium domain states of GMO. The detailed meaning of the figure is in the text. (b) Figure illustrating the simultaneous creation of a FF domain wall (green hatched line I-II) and annihilation of an antiphase domain wall (red hatched line III-I) in GMO. (c) Cloverleaf domain pattern configuration observed in $\mathrm{YMnO}_{3}$ [31]. The six ferroelectric domains of alternating up-down polarization (green-red) emerge from a single six-domain-state point, together with the annihilation of the domain walls between the three antiphase domains.
This is because the order-parameter space has the point-group symmetry 4 containing only rotations about an axis perpendicular to the order-parameter plane $\left(\eta_{1}, \eta_{2}\right)$ [30]. Hence, this plane has no specific symmetry directions, the sums $\vec{V}_{\mathrm{I}}+\vec{V}_{\mathrm{II}}$, $\vec{V}_{\mathrm{II}}+\vec{V}_{\mathrm{III}}, \vec{V}_{\mathrm{III}}+\vec{V}_{\mathrm{IV}}, \vec{V}_{\mathrm{IV}}+\vec{V}_{\mathrm{I}}$ associated with the domain walls having the same orthorhombic symmetry as the domain states. The symmetry of the antiphase domain walls I-III and II-IV, given by $\vec{V}_{\mathrm{I}}+\vec{V}_{\mathrm{III}}=\vec{V}_{\mathrm{II}}+\vec{V}_{\mathrm{IV}}=(0,0)$, is the maximal isotropy subgroup $m_{x} m_{y} 2_{z}$ of the parent symmetry $\overline{4} m 2$.

The determination of the antiphase domain-wall symmetries is derived from the primary order-parameter space following the same summing rule as for ferroic domain walls. The antiphase domain walls in $\mathrm{LaAlO}_{3}, \mathrm{SrTiO}_{3}$, and GMO display the higher symmetry of the ferroelastic domains in which they form, due to the fact that they separate antiphase domains with opposite order-parameter components. However, this is not the most general case, and antiphase domain walls can have a lower symmetry than the domains they separate. Furthermore, the coexistence of ferroic and antiphase domain walls can lead to complex interactions that may result in the annihilation or creation of both types of domain walls for specific configurations of the domains [32]. For example, in GMO the junctions between the antiphase domain wall I-III and the FF domain wall III-II can merge at a triple point [Fig. 3(b)] corresponding to the annihilation of the antiphase wall I-III and the creation of the FF domain wall I-II, i.e., I-III + III-II $\rightarrow$ I-II. Reciprocally, the junctions between the FF domain walls I-II and II-III can produce an annihilation of the FF domain walls and creation of an antiphase domain wall I-III: I-II + II-III $\rightarrow$ I-III. A striking illustration of the annihilation-creation process is found in the vortexlike domain pattern of $\mathrm{YMnO}_{3}$ [31], which shows the existence of a six-domain-state point at which annihilation of the antiphase domains results in the creation of six adjacent ferroelectric domains [Fig. 3(c)]. The two-component order parameter associated with the cell-tripled $P 6_{3} / m m c(\vec{a}, \vec{b}, \vec{c}) \rightarrow P 6_{3} c m(2 \vec{a}+$ $\vec{b}, \vec{b}-\vec{a}, \vec{c})$ improper ferroelectric transition in $\mathrm{YMnO}_{3}$ gives rise to a total of six domain states combining three antiphase domains, resulting from the loss of the paraelectric translations $(\vec{a}, \vec{b})$, with two opposite ferroelectric $180^{\circ}$ domains along $\vec{c}$. The corresponding cloverleaf domain pattern contains alternating \pm and $\mp 180^{\circ}$ ferroelectric domain walls merging at a single point but no antiphase domain walls.

\section{SUMMARY AND CONCLUSION}

In summary, it has been shown on selected examples of transitions that the symmetries of ferroelectric, ferroelastic, and antiphase domain walls can be directly derived from the symmetry of the corresponding adjacent domains in the order-parameter vector space. In some cases, such as the walls between the $180^{\circ}$ domains in $\mathrm{BaTiO}_{3}$, one has to take into account a reduction of the parent order-parameter space. In all cases the domain-wall symmetry is an isotropy subgroup coinciding with the sum of the vectors associated with adjacent domains. Although only the point-group symmetry of the domain walls has been worked out, the procedure also provides the space-group symmetries of the domain walls from which one can deduce the Bravais lattice along the 
two crystallographic directions preserved by the orientation of the domain wall. In our illustrative examples, the maximal symmetry induced by the primary order parameter has been considered for the domain walls without taking into account specific constraints which may reduce their symmetry. Let us finally emphasize that our proposed theoretical approach of domain walls applies to higher-order ferroics, in which higherrank macroscopic tensors emerge spontaneously [33], or to nonferroic transitions [34] involving exclusively antiphase domains. By contrast, the domain-wall symmetry of magnetic ferroics and multiferroics will be described elsewhere, as it requires taking into account time-reversal symmetry.

The authors are grateful to B. Mettout for very helpful discussions. This work was supported by the National Research Fund, Luxembourg (FNR/P12/4853155/Kreisel). P.T. is grateful to the CRP Gabriel Lippmann for financial support during his stay as visiting scientist.
[1] V. K. Wadhawan, Introduction to Ferroic Materials (Gordon and Breach, Amsterdam, 2000).

[2] H. Bea and P. Paruch, Nature Mater. 8, 168 (2009).

[3] E. K. H. Salje, Chem. Phys. Chem. 11, 940 (2010).

[4] G. Catalan, J. Seidel, R. Ramesh, and J. F. Scott, Rev. Mod. Phys. 84, 119 (2012).

[5] A. Aird, M. C. Domeneghetti, F. Mazzi, V. Tazzoli, and E. K. H. Salje, J. Phys.: Condens. Matter 10, L569 (1998).

[6] J. Seidel, L. W. Martin, Q. He, Q. Zhan, Y.-H. Chu, A. Rother, M. E. Hawkridge, P. Maksymovych, P. Yu, M. Gajek et al., Nature Mater. 8, 229 (2009).

[7] T. Sluka, A. K. Tagantsev, P. Bednyakov, and N. Setter, Nat. Commun. 4, 1808 (2013).

[8] M. Schröder, A. Haußmann, A. Thiessen, E. Soergel, T. Woike, and L. M. Eng, Adv. Funct. Mater. 22, 3936 (2012).

[9] S. Van Aert, S. Turner, R. Delville, D. Schryvers, G. Van Tendeloo, and E. K. H. Salje, Adv. Mater. 24, 523 (2012).

[10] E. K. H. Salje, O. Aktas, M. A. Carpenter, V. V. Laguta, and J. F. Scott, Phys. Rev. Lett. 111, 247603 (2013).

[11] V. Janovec and J. Přívratská, International Tables for Crystallography, edited by A. Authier (Wiley, New York, 2006), Vol. D, Chap. 3.4, pp. 449-505.

[12] J. Erhart, Phase Transitions 77, 989 (2004).

[13] A. A. Bul'bich and Y. M. Gufan, Sov. Phys. JETP 67, 1153 (1988).

[14] J. Lajzerowicz and J. J. Niez, J. Phys. (Paris) 40, L (1979).

[15] E. B. Sonin and A. K. Tagantsev, Ferroelectrics 98, 291 (1989).

[16] D. Lee, R. K. Behera, P. Wu, H. Xu, Y. L. Li, S. B. Sinnott, S. R. Phillpot, L. Q. Chen, and V. Gopalan, Phys. Rev. B 80, 060102(R) (2009).

[17] P. V. Yudin, A. K. Tagantsev, E. A. Eliseev, A. N. Morozovska, and N. Setter, Phys. Rev. B 86, 134102 (2012).
[18] V. Stepkova, P. Marton, and J. Hlinka, J. Phys.: Condens. Matter 24, 212201 (2012).

[19] A. V. Zalesskii, A. M. Savvinov, I. S. Zheludev, and A. N. Ivashchenko, Sov. Phys. JETP 41, 723 (1975).

[20] A. N. Bogdanov, V. A. Galushko, V. T. Telepa, and D. A. Yablonskii, JETP Lett. 40, 1279 (1984).

[21] E. G. Galkina, E. A. Zavadskii, V. I. Kamenev, A. L. Sukstanskii, and D. A. Yablonskii, Sov. Phys. Solid State 28, 954 (1986).

[22] M. E. Lines and A. M. Glass, Principles and Applications of Ferroelectrics (Clarendon Press, Oxford, 1977).

[23] H. T. Stokes and D. M. Hatch, Isotropy Subgroups of the 230 Crystallographic Space Groups (World Scientific, Singapore, 1988).

[24] P. Tolédano and V. Dmitriev, Reconstructive Transitions in Crystals and Quasicrystals (World Scientific, Singapore, 1996).

[25] W. J. Merz, Phys. Rev. 95, 690 (1954).

[26] D. S. Keeble and P. A. Thomas, J. Appl. Crystallogr. 42, 480 (2009).

[27] S. Bueble, K. Knorr, E. Brecht, and W. W. Schmahl, Surf. Sci. 400, 345 (1998).

[28] J. F. Scott, Phys. Rev. 183, 823 (1969).

[29] L. E. Cross, A. Fouskova, and S. E. Cummins, Phys. Rev. Lett. 21, 812 (1968).

[30] J.-C. Tolédano and P. Tolédano, The Landau Theory of Phase Transitions (World Scientific, Singapore, 1987).

[31] S. C. Chae, Y. Horibe, D. Y. Jeong, S. Rodan, N. Lee, and S.-W. Cheong, Proc. Natl. Acad. Sci. USA 107, 21366 (2010).

[32] V. A. Meleshina, V. L. Indenbom, K. S. Bagdasarov, and T. M. Polkhovskaya, Sov. Phys. Crystallogr. 18, 764 (1974).

[33] P. Tolédano and J.-C. Tolédano, Phys. Rev. B 16, 386 (1977).

[34] P. Tolédano and J.-C. Tolédano, Phys. Rev. B 25, 1946 (1982). 